ESTUDIOS / RESEARCH STUDIES

\title{
Indicadores de actividad tecnológica por género en España a través del estudio de patentes europeas
}

\author{
Elba Mauleón*, María Bordons* \\ *Departamento de Ciencia, Tecnología y Sociedad (CTS) \\ Instituto de Filosofía, Centro de Ciencias Humanas y Sociales (CCHS) \\ Consejo Superior de Investigaciones Científicas (CSIC) \\ Madrid, España \\ Correo-e: maria.bordons@cchs.csic.es; elba114@hotmail.com
}

Recibido: 05-06-2013; 2a version: 11-12-2013; Aceptado: 02-01-2014.

Cómo citar este artículo/Citation: Mauleón, E.; Bordons, M. (2014). Indicadores de actividad tecnológica por género en España a través del estudio de patentes europeas. Revista Española de Documentación Científica, 37(2):e043. doi: http://dx.doi. org/10.3989/redc.2014.2.1093

Resumen: El interés por los indicadores de actividad tecnológica por género responde a la necesidad de conocer cuál es la contribución de las mujeres a la innovación y al desarrollo tecnológico, actividades que son esenciales para el desarrollo económico de los países y en las que las mujeres han estado tradicionalmente poco representadas. Este trabajo asume una aproximación "macro" y presenta indicadores de actividad tecnológica por género basados en el análisis de las patentes solicitadas por inventores españoles en la Oficina Europea de Patentes (EPO) en el periodo 1999-2007. Se identifican un total de 6.860 solicitudes de patente con todos sus inventores españoles, el $20 \%$ de las cuales presenta participación femenina. Se observan diferencias por género entre sectores institucionales, con una mayor actividad femenina en el sector público de investigación; y por campos tecnológicos, con una mayor presencia de las mujeres en el área de Química. Se explora la relación entre el sexo de los inventores, el sector institucional del solicitante y el campo tecnológico de la patente a través de un análisis de correspondencias. Se señala el interés de los indicadores de patentes desagregados por género para identificar situaciones de desequilibrio por género en la actividad tecnológica y apoyar la implementación de políticas orientadas a favorecer la integración de la mujer investigadora en el sistema de ciencia y tecnología.

Palabras clave: Indicadores de ciencia y tecnología; patentes; innovación; mujer y ciencia; género.

\section{Gender-based indicators for technological activity in Spain based on the study of European patents}

Abstract: Obtaining indicators of technological activity by gender is needed to increase our knowledge about the contribution of women to innovation and technological development. These activities are essential for the economic success of countries but have traditionally revealed a low presence of females. This paper adopts a "macro" approach and presents technology indicators by gender based on the analysis of the patents applied for from the European Patent Office (EPO) by Spanish inventors during 1999-2007. From a total of 6,860 patents, at least one female inventor was found in $20 \%$ of the patents. Inter-gender differences in patenting are observed by institutional sector, with female involvement being above average in the public research sector; and by technological field, with a higher presence of females in chemistry. The relationship among the sex of the inventor, the institutional sector of the assignee and the technological field of the patent is explored through correspondence analysis. The article points out the relevance of patent-based indicators by gender for identifying gender inequalities in technological activity and supporting policy measures oriented to promote women's integration in the science and technology system.

Keywords: Science and technology indicators; patents; innovation; women in science; gender.

Copyright: (c) 2014 CSIC. Este es un artículo de acceso abierto distribuido bajo los términos de la licencia Creative Commons Attribution-Non Commercial (by-nc) Spain 3.0. 


\section{INTRODUCCIÓN}

La infra-representación de las mujeres en la ciencia es un hecho ampliamente descrito en la mayor parte de los países. En la Unión Europea, las mujeres constituyen un tercio de los investigadores en los centros de investigación gubernamentales y en la universidad, mientras que su presencia desciende al $15 \%$ en el entorno empresarial. Estas cifras varían de unos países a otros, pero un factor común a todos ellos es la denominada "segregación horizontal", según la cual las mujeres tienden a concentrarse en determinadas áreas temáticas, y la "segregación vertical", que se refiere a la menor presencia femenina al ascender en la jerarquía profesional. En este contexto, diversos organismos internacionales señalan la necesidad de promover la integración de las mujeres en la ciencia, no solo para defender los derechos de las mujeres, sino también para evitar el mal uso de los recursos que supone su no integración (Comisión Europea, 2001).

En el marco de la Unión Europea, la Comisión ha remarcado el interés de trabajar simultáneamente en varias direcciones, como son: obtener indicadores desagregados por género; identificar y analizar los factores que generan desigualdades por género; y establecer mecanismos para promover la integración de las mujeres en la ciencia (Dewandre, 2002). El interés de los indicadores desagregados por género es evidente, ya que es el punto de partida para el desarrollo del resto de acciones. La obtención de forma rigurosa y sistemática de datos sobre la situación de la mujer en la ciencia y la tecnología es esencial para poder realizar un seguimiento a lo largo del tiempo, analizar la eficacia de las medidas implementadas y realizar comparaciones entre regiones y países. Sobre esta base se creó en 1999 el "Grupo de Helsinki", con el fin de reunir estadísticas sobre la situación de las mujeres en ciencia en los distintos países europeos y para promover el debate y el intercambio de experiencias entre países. Un primer resultado fue el Informe ETAN (Comisión Europea, 2000), que ofrecía una primera panorámica de la situación de las mujeres en la ciencia europea. Posteriormente, la Comisión ha financiado otros estudios, resumidos sintéticamente en la publicación Stocktaking 10 years of "Women in Science", de 2010, que profundizan en distintos aspectos del problema y difunden recomendaciones para facilitar la integración de las mujeres en la ciencia. Especial mención merece el informe She Figures, que se publica desde 2003 cada tres años incluyendo estadísticas sobre la situación de las mujeres en los distintos países de la Unión Europea (última edición en 2013) (Comisión Europea, 2013).

Distintos estudios señalan una mayor brecha de género en tecnología que en ciencia, lo que resulta preocupante porque la innovación y la creación de nuevo conocimiento tecnológico es un motor básico del crecimiento económico de los países.
Para maximizar este crecimiento cada país tiene que contar con la capacidad innovadora de todos sus recursos humanos, independientemente de su sexo, por lo que conocer y fomentar la participación de las mujeres en este tipo de actividades adquiere especial relevancia. Dado que las patentes constituyen un indicador de actividad tecnológica ampliamente aceptado, cuyas ventajas y limitaciones están repetidamente recogidas en la literatura (Nesta y Patel, 2004), es indudable el interés de analizar la presencia de hombres y mujeres entre los inventores de patentes para incrementar nuestro conocimiento sobre su participación en los procesos de innovación y de creación de nuevo conocimiento tecnológico.

Existen distintos estudios en la literatura que exploran las posibles diferencias por género en la actividad tecnológica a través del análisis de patentes. Predominan los estudios que recogen información a nivel micro partiendo de una muestra de investigadores en determinados centros o áreas temáticas, recogen su participación como inventores de patentes y exploran diferencias por género en distintos aspectos como son la tendencia a patentar, el número de patentes por investigador o el impacto de las invenciones (Morgan y otros, 2001; Whittington y Smith-Doerr 2005; Ding y otros, 2006; Azoulay y otros, 2007; Hunt y otros, 2013). Estos estudios han puesto de manifiesto una menor tendencia de las mujeres a patentar tanto en el contexto académico como en la industria (Whittington and Smith-Doerr, 2005); han identificado algunos factores que explican estas diferencias a través de entrevistas con investigadores; y han explorado su influencia mediante modelos econométricos (Ding y otros, 2006; Murray y Graham, 2007; Hunt y otros, 2013).

Por otro lado, los estudios a nivel macro, que se centran en el estudio de la actividad tecnológica de países y descienden a explorar posibles diferencias por sectores institucionales y campos, son limitados. La mayor dificultad radica en identificar el sexo de los inventores, ya que es una variable que no recogen las solicitudes de patentes y que hay que obtener de fuentes complementarias o inferir a partir del nombre de los inventores. El estudio de Naldi (Naldi y otros, 2004), financiado por la Comisión Europea, fue pionero en analizar la posibilidad de producir indicadores basados en publicaciones y patentes por género en una muestra de países europeos (Alemania, España, Francia, Italia, Reino Unido y Suecia), observando que el $97 \%$ de las patentes solicitadas por dichos países a la Oficina Europea de Patentes en 1998 tenían al menos un inventor masculino, frente al $12 \%$ que incluía alguna inventora. Posteriormente, Frietsch y colaboradores (Frietsch y otros, 2009) analizaron las publicaciones y patentes solicitadas en la Oficina Europea de Patentes (EPO) por 14 países durante un periodo de 5 años, revelando notables diferencias por países en la participación femenina, menor en los países de Europa central (p.ej. Alemania o 
Suiza) y mayor en los países mediterráneos (España, Francia, Italia) y poniendo de manifiesto una mayor participación femenina en ciencia (publicaciones) que en tecnología (patentes). El estudio de Busolt y Kugele (2009) analizó las patentes solicitadas a la EPO por algún país miembro de la UE-27 durante 2001-2003 y mostró que sólo un $8 \%$ de los inventores eran mujeres. Estos autores señalaron la menor presencia de mujeres inventoras en los países con una sistema nacional de innovación más desarrollado; la tendencia de las mujeres a concentrarse en el sector público frente al privado; y su preferencia por determinados campos científicos como química y farmacia.

Aunque los estudios anteriores ponen de manifiesto el interés de la obtención periódica de indicadores desagregados por género basados en patentes para realizar un seguimiento a lo largo del tiempo de la implicación de hombres y mujeres en la actividad inventiva, las oficinas de patentes no proporcionan habitualmente estos indicadores por las dificultades derivadas de la identificación del sexo de los inventores. Por esta razón, la mayor parte de los estudios existentes han sido realizados en el marco de contratos (ver por ejemplo, Delixus 2012) o proyectos de investigación (Naldi y otros, 2004; Busolt y Kugele, 2009). En lo que se refiere a España, la Oficina Española de Patentes y Marcas no desagrega sus estadísticas por sexo, pero proporciona algunos datos de presencia femenina entre los titulares de las patentes, de forma que un $8 \%$ de las patentes solicitadas entre 1964 y 2008 contaban con alguna mujer, cifra que asciende al $10 \%$ en los últimos años del periodo (OEPM 2009). No obstante, hay que tener en cuenta que dicho análisis se centra en las patentes solicitadas por particulares, que constituyen el $48 \%$ del total, excluyendo las solicitadas por organismos o empresas en las que no suele figurar una persona física como solicitante. Un trabajo posterior que analiza las patentes solicitadas a la Oficina Española de Patentes y Marcas (OEPM) durante 1990-2005 (Mauleón y Bordons, 2010) atendiendo al sexo de los inventores evidencia una baja actividad femenina, que tendió a incrementarse a lo largo de los años ( $8 \%$ de inventoras en 1990 frente a un $15 \%$ en 2005).

El objetivo de este estudio es analizar la participación de las mujeres en el desarrollo tecnológico en España medido a través de su actividad como inventoras en las patentes solicitadas en la Oficina Europea de Patentes. Este estudio se enmarca en una línea de trabajo orientada a la obtención de indicadores bibliométricos por género y presenta algunos avances metodológicos respecto a estudios previos sobre patentes españolas (Mauleón y Bordons, 2010; Mauleón y otros, 2014). Por un lado, las patentes europeas se suponen de mayor valor económico que las solicitadas en la Oficina Española, dado el mayor coste de proteger una patente a nivel internacional. Por otro lado, se han identificado las patentes con todos sus inventores espa- ñoles, para evitar el posible sesgo originado por la inclusión de inventores extranjeros. Además, se ha utilizado la clasificación por campos tecnológicos realizada en colaboración entre el Fraunhofer ISI (Fraunhofer Institute for Systems and Innovation Research, Karlsruhe-Alemania), OST (Observatoire des Sciences et des Techniques, Paris-Francia) e INPI (Institut National de la Propriété Industrielle, Francia), en lugar de la Clasificación Internacional de Patentes (CIP), por considerar que ésta última presenta limitaciones al responder sobre todo a criterios administrativos, mientras que la clasificación Fraunhofer ISI/OST/INPI se ajusta mejor al desarrollo de la ciencia y la tecnología actual. Por último, se explora la utilidad del análisis de correspondencias para analizar y visualizar la relación entre el género de los inventores y otras variables.

\section{OBJETIVOS}

Se estudia la actividad investigadora española en su vertiente tecnológica desde una perspectiva de género a través del análisis de las patentes con inventores españoles solicitadas a la Oficina Europea de Patentes durante 1999-2007. Se desea conocer la presencia de hombres y mujeres entre los inventores de patentes; explorar posibles diferencias por género en su especialización temática, sector institucional solicitante de la patente y hábitos de colaboración; y observar si aumenta la actividad femenina a lo largo del periodo en estudio. Finalmente, se desea visualizar de forma conjunta la asociación entre las variables sexo, sector institucional y campo tecnológico mediante el análisis de correspondencias.

Con este estudio pretendemos señalar la importancia y el interés de obtener indicadores de actividad tecnológica por género, tal y como sugieren las recomendaciones formuladas por la UE (Comisión Europea, 2010). Se describen algunas dificultades y limitaciones en la obtención de este tipo de indicadores y se muestran tendencias en la implicación de hombres y mujeres en la actividad inventiva de España.

\section{METODOLOGÍA}

Este trabajo analiza la actividad tecnológica por género en España a través del estudio de las patentes con inventores españoles solicitadas a la Oficina Europea de Patentes (EPO). De esta forma, la unidad de estudio en esta investigación es la solicitud de patentes publicada, accesible al público a través de la base de datos esp@cenet. Se consideran patentes solicitadas, tanto si han sido concedidas o no, porque esta investigación está dirigida a estudiar la implicación de hombres y mujeres en la actividad tecnológica, independientemente del éxito final de la patente, es decir, de si se concede y de si se llega a licenciarse para su explotación comercial. No obstante, el porcentaje de patentes concedidas es muy alto, cerca del $80 \%$ en el caso 
de las patentes europeas, de manera que el uso de las solicitudes de patentes para estudiar la capacidad innovadora es adecuado para describir la participación de hombres y mujeres en innovación. Por último, hay que señalar que en el estudio se ha tomado como referencia la fecha de solicitud de la patente; esto es algo muy habitual en los estudios de patentes porque representa la primera comunicación formal de una invención (Shild 1999; Balconi y otros, 2004; Breschi y otros, 2004) y se liga de forma más directa con el momento en que se realizó la invención.

Las patentes con inventores españoles solicitadas a la Oficina Europea de Patentes en el periodo 1999-2007 se descargaron de la base de datos esp@cenet y se creó una base de datos relacional para su posterior tratamiento y normalización. Esta base de datos incluía todos los inventores y solicitantes de cada patente, junto con su país, e información bibliográfica relativa a las patentes (fecha de solicitud, código de la clasificación internacional de patentes, título de la patente, etc).

El inventor de la patente es quien desarrolla la idea y puede ser un individuo o varios, en cuyo caso estaríamos hablando de co-inventores. El solicitante de una patente es quien asume los gastos de solicitud y mantenimiento de la patente y en la mayoría de los casos suele tratarse de una institución (universidades, organismos de investigación, empresas, firmas comerciales, etc.), pero también de individuos que solicitan la patente a título personal. En definitiva, el inventor se refiere a quién produce la invención y el solicitante a quién explota económicamente el conocimiento patentado.

a) Normalización de los datos relativos al solicitante

En las bases de datos de patentes, la información relativa a los solicitantes no está normalizada, por lo que una institución puede quedar registrada bajo nombres diferentes, entorpeciendo el estudio de la producción a nivel institucional. Para evitar este problema, se codificaron las instituciones solicitantes siguiendo una metodología propia para el tratamiento y codificación de los lugares de trabajo en las publicaciones (Morillo y otros, 2013).

Atendiendo al tipo de solicitante, se han agrupado las patentes según la siguiente clasificación: CSIC, Empresa, Extranjero, Particular, Universidad y Otros. Dentro de "Otros" se incluyen sectores con baja orientación a patentar como son la administración, el sector sanitario o las entidades sin ánimo de lucro.

En este trabajo se ha utilizado el método de recuento total, de forma que si dos o más sectores institucionales aparecen como solicitantes de una patente, ésta se ha asignado a todos y cada uno de ellos.

\section{b) Identificación del sexo del inventor}

La asignación del sexo se realizó en base al nombre completo de los inventores, bien por estar recogido en la base de datos de patentes, o por obtenerse externamente en el proceso de identificación de los inventores a través de búsquedas en Internet. A diferencia de lo que ocurre con las publicaciones científicas, en las que muchas veces sólo se incluye la inicial del nombre del autor seguida de su/s apellido/s, en el caso de las patentes es habitual que se registre el nombre completo de los inventores y sus dos apellidos. En este estudio se identificó el sexo de todos los inventores en el $99 \%$ de las patentes.

\section{c) Clasificación temática de patentes}

El campo tecnológico al que pertenece cada patente se indica a través de los códigos asignados por expertos según la Clasificación Internacional de Patentes (CIP), que es un sistema jerárquico utilizado a nivel internacional. Esta clasificación se basa sobre todo en criterios técnicos, está muy alejada de las clasificaciones que se utilizan en la caracterización de las actividades económicas, y no refleja bien los campos emergentes. Para solventar estas limitaciones, el Fraunhofer ISI, el Observatoire des Sciences et des Technologies (OST) y el Institut National de la Propriété Industrielle (INPI) desarrollaron en 1992 una clasificación basada en una re-agrupación de las categorías de la CIP, que se ha actualizado posteriormente de forma periódica. En este trabajo se ha utilizado la tabla de correspondencias OST/ INPI/ISI (OMPI, 2008; Schmoch, 2008) que permite la asignación de patentes a campos tecnológicos, favoreciendo así la utilización de la patente como un indicador del proceso de innovación.

Además, se analiza la presencia de hombres y mujeres en las patentes de tres áreas concretas: Biotecnología, Tecnologías de la Información y la Comunicación (TICs) y Alta Tecnología. El interés de analizar la contribución de las mujeres en estos campos radica en que son sectores que por su complejidad requieren un importante esfuerzo investigador y una sólida base tecnológica, son determinantes para la competitividad de un país y su desarrollo tiene fuertes implicaciones y efectos en la economía y la sociedad. Estos campos se han delimitado siguiendo los criterios de Eurostat (http:// epp.eurostat.ec.europa.eu/cache/ITY_SDDS/Annexes/pat_esms_an4.pdf).

\section{d) Indicadores de actividad por género}

Se han utilizado los indicadores propuestos por Naldi (Naldi y otros, 2004), que incluyen:

\footnotetext{
- Participación: porcentaje de patentes cuyos inventores son "sólo hombres", "sólo mujeres" o equipos mixtos. Se calcula también el porcentaje de patentes con "alguna mujer", que incluye las dos últimas categorías.
} 
- Contribución: se basa en un recuento fraccionado. En una patente con $n$ inventores, la contribución de cada género es el número de inventores de dicho género dividido por " $n$ ", de forma que la suma de la contribución de ambos géneros será 1 . Oscila entre 0 y 1 , aunque en este estudio se considera en porcentaje (rango de 0-100\%).

- Presencia: es el porcentaje de hombres y mujeres entre los inventores de las patentes. La presencia considera todos los inventores por igual, independientemente de que aparezcan como inventores únicos o como co-inventores. Por el contrario, en el cálculo de la contribución se tiene en cuenta el número de inventores de cada patente, de forma que si una mujer colabora con tres hombres en una patente se contabilizará 0,25 para el sexo femenino y 0,75 para el masculino.

Para analizar posibles diferencias por género en la tendencia de los inventores a colaborar se ha analizado la presencia, contribución y participación femenina en función del número de inventores de las patentes.

Este estudio se centra en las patentes con todos sus inventores españoles, ya que nuestro interés es obtener indicadores de actividad tecnológica por género en nuestro país y la inclusión de inventores extranjeros podría distorsionar los resultados.

\section{e) Análisis de correspondencias múltiple}

El Análisis de Correspondencias es una técnica estadística multivariante que permite resumir una gran cantidad de información en un número reducido de dimensiones o factores, con la menor pérdida de información posible (Benzécri, 1973). El método se aplica sobre variables categóricas u ordinales. Permite poner de manifiesto gráficamente las relaciones de dependencia existentes entre las diversas modalidades de dos o más variables categóricas a partir de la información proporcionada por sus tablas de frecuencias cruzadas. Es decir, su ventaja es que no sólo analiza dependencia entre variables, sino que muestra qué modalidades de cada variable están más asociadas entre sí. Esta técnica ya ha sido aplicada anteriormente en análisis basados en patentes (ver por ejemplo Lascuraín y otros, 2010), pero en este estudio se aplica un análisis de correspondencias múltiple, porque se cuenta con más de dos variables nominales. En concreto, se analiza la relación existente entre el campo tecnológico de las patentes, el sector institucional del solicitante y la visibilidad femenina medida a través de la participación. El estudio se realiza mediante el programa SPSS (versión 19).

\section{RESULTADOS}

En el periodo 1999-2007 se presentaron en la Oficina Europea de Patentes un total de 8.623 solicitudes de patentes con algún inventor español. La mayor parte de las solicitudes provenían de la empresa $(60 \%)$, siendo mucho menor la actividad procedente de los particulares (19\%), la universidad (4\%) y el CSIC (3\%). En lo que respecta a los campos con mayor actividad tecnológica destacan Ingeniería Mecánica (39\%) y Química $(27 \%)$, seguidas de Ingeniería Eléctrica $(17 \%)$, Instrumentación (12\%) y Otros campos $(21 \%)$. A lo largo del periodo se observa una creciente colaboración en la actividad inventiva, incrementándose el número medio de inventores por patente desde 1,67 en 1999 hasta 2,20 en 2007 (43\% de patentes con un solo inventor en 1999 vs. $28 \%$ en 2007). Nuestro estudio desde una perspectiva de género se centra en 6.860 patentes con todos sus inventores españoles (80\% del total).

\subsection{Participación, contribución y presencia por} campos tecnológicos y sectores institucionales

En los años estudiados, apenas un 4\% de las patentes tenían todos sus inventores mujeres, frente a un $80 \%$ de patentes firmadas sólo por hombres y un $16 \%$ por equipos mixtos. La contribución femenina asciende al 10\%, y la presencia al 15\%.

No obstante, se observan diferencias en la implicación de hombres y mujeres por campos tecnológicos y sectores institucionales. La tabla I y la figura 1 muestran la alta especialización de las mujeres en el área Química, donde el porcentaje de patentes con alguna mujer es muy superior al detectado en el total de patentes (44\% vs $20 \%$ ). Aunque más del $40 \%$ de las patentes de Química cuentan con alguna mujer, estas tienden a colaborar con inventores masculinos lo que explica los menores valores alcanzados por la contribución femenina (recuento fraccionado). La figura 1 muestra que los equipos mixtos de inventores predominan sobre las patentes firmadas sólo por mujeres en prácticamente todos los campos.

En lo que se refiere a los sectores institucionales, la mayor actividad femenina se detecta en el sector público $y$, en concreto, en la universidad y en el CSIC (Tabla II). Los sectores con menor número de patentes son los que presentan mayor actividad femenina, mientras que la empresa, que lidera la actividad tecnológica por número de patentes, muestra baja participación de mujeres. En "Otros sectores" se incluyen los centros del sector sanitario y la administración pública (OPIs), con baja actividad inventiva, pero alta presencia femenina (Tabla II, Figura 1)

¿Existen diferencias en la tendencia de hombres y mujeres a colaborar? La figura 2 muestra la participación, contribución y presencia femenina según el número de inventores en las patentes, observándose una mayor visibilidad de las mujeres al aumentar el número de inventores. Así, considerando el indicador presencia femenina, en el total del periodo el porcentaje de mujeres en las pa- 
Tabla I. Participación, contribución y presencia por campos tecnológicos y género (EPO, 1999-2007)

\begin{tabular}{|c|c|c|c|c|c|c|c|c|}
\hline & \multicolumn{3}{|c|}{ Participación } & \multicolumn{2}{|c|}{ Contribución } & \multicolumn{3}{|c|}{ Presencia } \\
\hline & $\begin{array}{l}\text { \% Solo } \\
\text { hombres }\end{array}$ & $\begin{array}{l}\text { \%Alguna } \\
\text { mujer }\end{array}$ & $\begin{array}{c}\text { N. } \\
\text { Patentes }\end{array}$ & $\begin{array}{c}\% \\
\text { masculina }\end{array}$ & $\begin{array}{c}\% \\
\text { femenina }\end{array}$ & $\begin{array}{c}\% \\
\text { Hombres }\end{array}$ & $\begin{array}{c}\% \\
\text { Mujeres }\end{array}$ & $\begin{array}{c}\mathbf{N} . \\
\text { inventores }\end{array}$ \\
\hline Ingeniería Eléctrica & 84,44 & 15,56 & 1144 & 92,88 & 7,12 & 90,83 & 9,17 & 2333 \\
\hline Instrumentación & 81,68 & 18,32 & 819 & 91,41 & 8,59 & 87,66 & 12,34 & 1685 \\
\hline Química & 56,37 & 43,63 & 1820 & 78,77 & 21,23 & 72,57 & 27,43 & 5340 \\
\hline Ingeniería Mecánica & 89,87 & 10,13 & 2666 & 94,38 & 5,62 & 92,47 & 7,53 & 4397 \\
\hline Otros campos & 90,21 & 9,79 & 1409 & 92,69 & 7,31 & 92,52 & 7,48 & 2031 \\
\hline Total & 80,19 & 19,81 & 6816 & 89,68 & 10,32 & 84,99 & 15,01 & 13864 \\
\hline
\end{tabular}

Nota: el sumatorio del número de patentes e inventores es superior al total real porque algunas patentes están asignadas a más de un campo tecnológico. "Alguna mujer" incluye patentes con todos sus inventores mujerest y patentes con equipos mixtos.

Tabla II. Participación, contribución y presencia por sectores institucionales y género (EPO, 1999-2007)

\begin{tabular}{lccc|cc|ccc}
\hline & \multicolumn{3}{c}{ Participación } & \multicolumn{3}{c}{ Contribución } & \multicolumn{3}{c}{ Presencia } \\
\cline { 2 - 9 } & $\begin{array}{c}\text { \% Solo } \\
\text { hombres }\end{array}$ & $\begin{array}{c}\text { \% Alguna } \\
\text { mujer }\end{array}$ & $\begin{array}{c}\mathbf{N} \text {. } \\
\text { Patentes }\end{array}$ & $\begin{array}{c}\text { \% } \\
\text { Masc. }\end{array}$ & $\begin{array}{c}\text { \% } \\
\text { Fem. }\end{array}$ & $\begin{array}{c}\text { \% } \\
\text { Hombres }\end{array}$ & $\begin{array}{c}\text { \% } \\
\text { Mujeres }\end{array}$ & $\begin{array}{c}\text { N. } \\
\text { Inventores }\end{array}$ \\
\hline Empresas & 82,27 & 17,73 & 4043 & 91,38 & 8,62 & 85,65 & 14,35 & 8479 \\
\hline Particulares & 88,09 & 11,91 & 1360 & 90,14 & 9,86 & 89,89 & 10,11 & 1701 \\
\hline Extranjero & 75,32 & 24,68 & 1082 & 88,00 & 12,00 & 85,18 & 14,82 & 2436 \\
\hline Universidad & 42,21 & 57,79 & 263 & 75,11 & 24,89 & 70,77 & 29,23 & 1016 \\
\hline CSIC & 39,01 & 60,99 & 182 & 74,20 & 25,80 & 72,65 & 27,35 & 713 \\
\hline Otros & 44,21 & 55,79 & 95 & 79,04 & 20,96 & 76,82 & 23,18 & 343 \\
\hline Total & 80,19 & 19,81 & 6816 & 89,68 & 10,32 & 84,18 & 15,82 & 13864 \\
\hline
\end{tabular}

Nota: el sumatorio del número de patentes e inventores es superior al total real porque existe colaboración entre distintos sectores institucionales en algunas patentes. "Alguna mujer" incluye patentes con todos sus inventores mujeres y patentes con equipos mixtos.

Figura 1. Participación por a) campos tecnológicos; y b) sectores institucionales (EPO, 1999-2007)
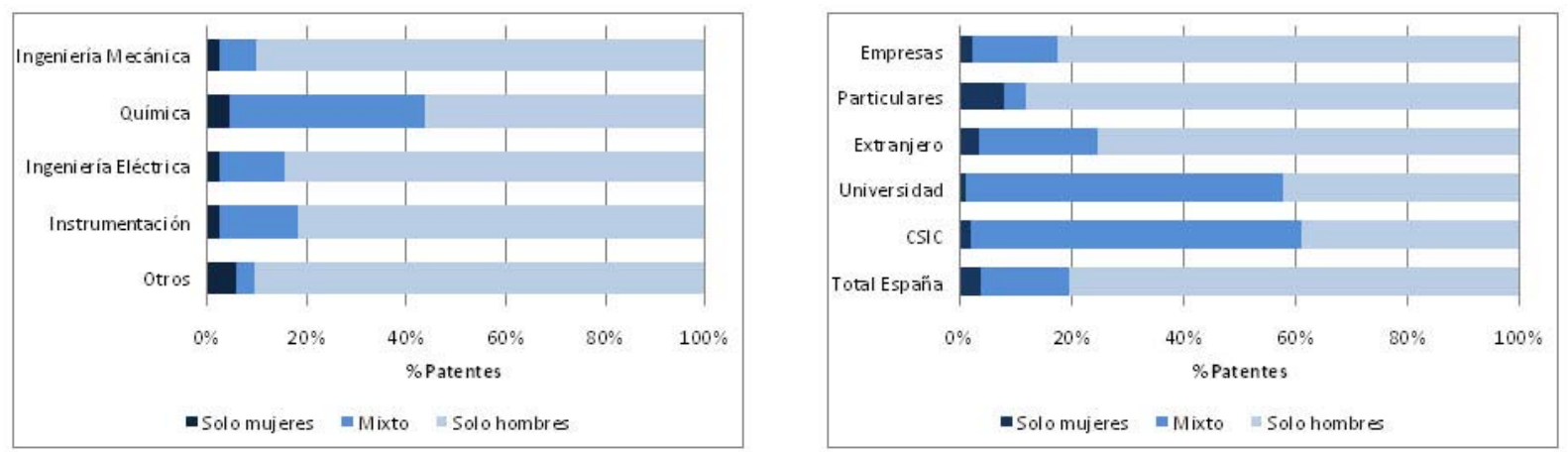
tentes con un solo inventor es del $5 \%$, frente al $19 \%$ en las patentes con más de un inventor. La misma tendencia se observa a través de la participación y de la contribución. Es interesante señalar que varios factores confluyen para explicar estos resultados. Por un lado, la mayor actividad de las mujeres en Química, área en la que los equipos son más numerosos (el $30 \%$ de las patentes de Química tienen un solo inventor frente al $65 \%$ en Ingeniería Mecánica) pero, además, incluso dentro de esta área, la visibilidad femenina se incrementa con el número de inventores, de forma que en las patentes con 1 solo inventor un $5 \%$ de los mismos son mujeres y entre las patentes con 2 o más inventores el porcentaje de mujeres es de un $30 \%$.

En el periodo analizado, las patentes en Alta Tecnología, Biotecnología y TIC constituyen el $10 \%$, $4 \%$ y $13 \%$ respectivamente del total de las patentes españolas con todos sus inventores españoles

Figura 2. Participación, contribución y presencia femenina según el número de inventores (EPO, 1999-2007)

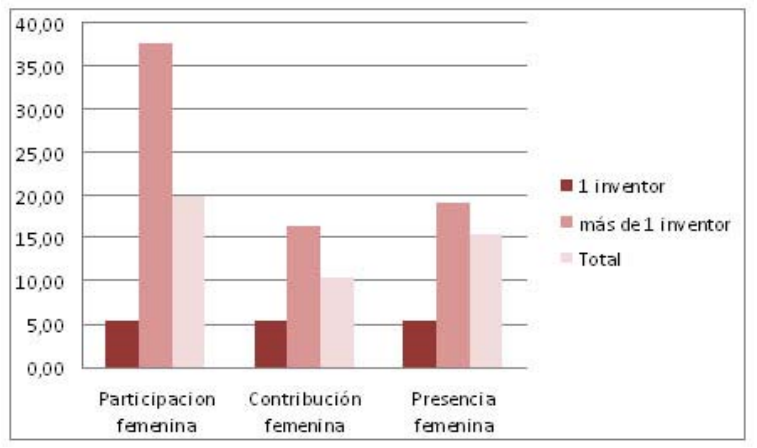

en el periodo en estudio. La mayor actividad femenina se detecta en Biotecnología, donde la participación, contribución y presencia femenina es muy superior a la media (Figura 3). La visibilidad femenina es similar a la media descrita para el conjunto de las áreas en Alta Tecnología, mientras que se sitúa por debajo en TIC.

\subsection{Evolución temporal de los indicadores por género}

A lo largo de los años se observa que aumenta la participación femenina, de un $16 \%$ a un $24 \%$ (patentes mixtas y patentes con todos sus inventores mujeres), la contribución de un $10 \%$ a un $13 \%$ y la presencia de un $12 \%$ a un $19 \%$ (Tabla III). Hay que señalar que el incremento observado es muy pequeño en todos los casos, y se produce sobre todo el último año del periodo analizado (Figura 4).

Figura 3. Visibilidad femenina en las patentes de alta tecnología (EPO, 1999-2007)

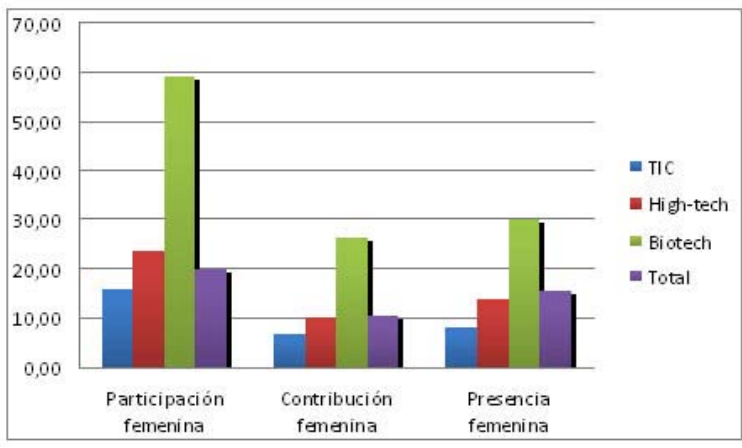

Tabla III. Participación, contribución y presencia de inventores según su sexo

\begin{tabular}{|c|c|c|c|c|c|c|c|c|c|}
\hline & \multicolumn{4}{|c|}{ Participación } & \multicolumn{2}{|c|}{ Contribución } & \multicolumn{3}{|c|}{ Presencia } \\
\hline & $\begin{array}{l}\text { \% Sólo } \\
\text { mujeres }\end{array}$ & $\begin{array}{c}\% \\
\text { Mixto }\end{array}$ & $\begin{array}{l}\text { \% Sólo } \\
\text { hombres }\end{array}$ & $\begin{array}{l}\text { N.Total } \\
\text { patentes }\end{array}$ & $\begin{array}{c}\% \\
\text { Masc }\end{array}$ & $\begin{array}{c}\% \\
\text { Fem }\end{array}$ & $\begin{array}{c}\% \\
\text { Hombres }\end{array}$ & $\begin{array}{c}\% \\
\text { Mujeres }\end{array}$ & $\begin{array}{c}\text { N.Total } \\
\text { inventores }\end{array}$ \\
\hline 1999 & 5,71 & 10,27 & 84,02 & 438 & 89,78 & 10,22 & 87,84 & 12,16 & 732 \\
\hline 2000 & 7,58 & 13,36 & 79,06 & 554 & 87,21 & 12,79 & 85,34 & 14,66 & 1003 \\
\hline 2001 & 3,28 & 12,30 & 84,43 & 610 & 91,91 & 8,09 & 88,39 & 11,61 & 1171 \\
\hline 2002 & 3,51 & 16,49 & 80,00 & 655 & 89,28 & 10,72 & 83,83 & 16,17 & 1299 \\
\hline 2003 & 3,69 & 15,93 & 80,38 & 678 & 90,14 & 9,86 & 83,55 & 16,45 & 1416 \\
\hline 2004 & 2,88 & 16,79 & 80,34 & 834 & 90,18 & 9,82 & 84,94 & 15,06 & 1726 \\
\hline 2005 & 3,01 & 14,72 & 82,27 & 863 & 91,24 & 8,76 & 86,76 & 13,24 & 1805 \\
\hline 2006 & 3,04 & 17,24 & 79,73 & 1021 & 90,41 & 9,59 & 84,88 & 15,12 & 2149 \\
\hline 2007 & 3,87 & 20,29 & 75,84 & 1163 & 87,43 & 12,57 & 80,80 & 19,20 & 2563 \\
\hline Total & 3,83 & 15,98 & 80,19 & 6816 & 89,68 & 10,32 & 84,63 & 15,37 & 13864 \\
\hline
\end{tabular}


¿Se incrementa la actividad femenina de forma particular en algún sector institucional o campo tecnológico? En lo que se refiere a los sectores institucionales, el mayor incremento de la contribución femenina se observa en la universidad ( 6 puntos porcentuales), otros centros (5 puntos) y el CSIC (4 puntos), seguido por el sector empresa (2 puntos porcentuales), mientras que las inventoras pierden visibilidad entre las patentes con solicitantes particulares o de centros extranjeros (se reduce la contribución en 4 y 3 puntos respectivamente) (Figura 4a). En lo que respecta a los campos tecnológicos, el mayor incremento en la contribución femenina se detecta en Instrumentación (3 puntos porcentuales) (Figura 4b).

Por último, hay que señalar que a lo largo del periodo se refuerza la tendencia de las mujeres a inventar de forma colectiva. A modo de ejemplo se puede mencionar que la contribución femenina en solitario desciende del $11 \%$ en el primer trienio del periodo a un $7 \%$ en el último trienio.

\subsection{Análisis de correspondencias}

Con el fin de analizar la relación existente entre el campo tecnológico de las patentes (cinco campos), el sector institucional del solicitante (seis sectores institucionales) y la visibilidad femenina medida a través de la participación femenina (tres categorías: sólo hombres, sólo mujeres y mixtas) se realiza un análisis de correspondencias. Aunque las tablas mostradas en la sección 4.1 ponen de manifiesto qué campos tecnológicos o sectores institucionales contribuyen más a explicar las diferencias entre sexos, el análisis de correspondencias permite profundizar en el conocimiento de las relaciones entre las variables y revelar la dependencia entre las distintas categorías de las variables. El estudio se limita al conjunto de patentes con un solo sector institucional y una sola clasificación temática, que asciende a 5667 patentes ( $83 \%$ del total).

El modelo resultante explica el $97 \%$ de la varianza explicada con dos factores o dimensiones. A través de la dimensión 1 se explica el $55,1 \%$ de la varianza total y a través de la dimensión 2 , se explica el 41,8\%. En la dimensión 1 todas las variables presentan buenos valores de discriminación, es decir, que todas contribuyen a definir este factor o dimensión, aunque las que más discriminan son campo tecnológico y sexo. En la dimensión 2 las variables que más discriminan son campo tecnológico y sector (tabla IV).

Figura 4. Evolución de la contribución femenina según a) sectores institucionales y b) campos tecnológicos
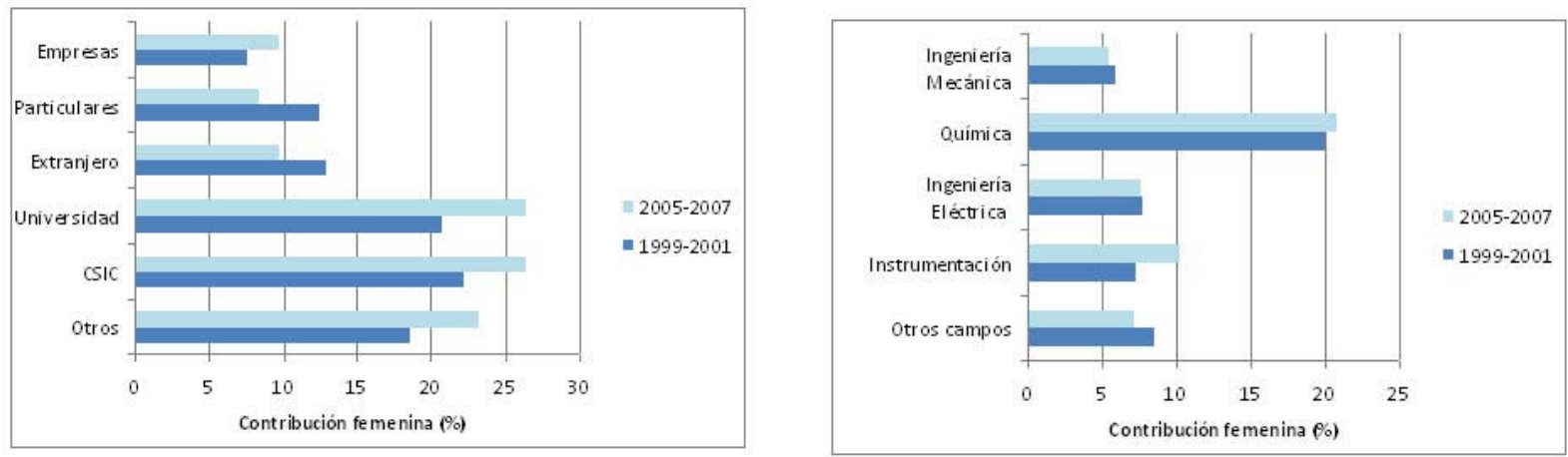

Tabla IV. Resultado del análisis de correspondencias

\begin{tabular}{lccc}
\hline \multicolumn{2}{c}{ Medidas de discriminación } \\
\hline & \multicolumn{2}{c}{ Dimensión } & Media \\
\cline { 2 - 4 } & 1 & 2 &, 512 \\
\hline Sector institucional &, 448 &, 576 &, 333 \\
\hline Participación por sexo &, 575 &, 092 &, 607 \\
\hline Campo tecnológico &, 630 &, 585 & 1,453 \\
\hline Total activo & 1,653 & 1,253 & \\
\hline
\end{tabular}


Con el fin de facilitar la adecuada interpretación del análisis de correspondencias, es importante recordar que en este análisis a cada categoría o modalidad de una variable se le asocia un punto en el espacio de forma que, cuanto más alejado del origen de coordenadas está el punto, mejor está representado, y cuanto más cercanas estén las categorías entre sí, mayor asociación hay entre ellas. Por otro lado, si dos categorías de variables diferentes están cercanas es que están presentes en una misma patente. Si dos categorías de una misma variable están próximas, es porque las patentes que las poseen presentan casi el mismo comportamiento en las otras variables. Finalmente, sólo mencionar que las categorías con pocos casos tienden a estar más alejadas del centro de gravedad.

Tal y como se observa en la figura 5 , el eje 1 separa las patentes mixtas (a la izquierda del eje), de aquellas con sólo hombres o sólo mujeres (a la derecha del eje), es decir, que discrimina según los equipos tengan inventores de diferente sexo o de solo un solo sexo. El eje 2 separa fundamentalmente las patentes de solicitante extranjero y de Ingeniería Eléctrica de las de los demás sectores institucionales y campos tecnológicos. La cercanía entre solicitante extranjero e Ingeniería Eléctrica indica que existe afinidad entre ambas categorías, es decir, que los solicitantes extranjeros muestran alta actividad en este campo.

Podemos ver que el CSIC, la Universidad y Otros sectores están próximos entre sí, lo que indica que tienen un comportamiento similar. Se observa una asociación positiva entre estos sectores, las patentes con equipos mixtos de inventores y el campo de la Química. Por otra parte, las patentes de particulares están más cercanas a las categorías "solo hombres" y "solo mujeres", lo que es compatible con el alto porcentaje de patentes con un solo inventor entre los particulares $(80 \%)$. Destaca la proximidad entre "solo hombres" e Ingeniería Mecánica, lo que indica una fuerte especialización masculina en este campo.

La empresa se sitúa en el centro de coordenadas porque es la categoría de mayor tamaño, y su situación hace que no quede bien explicada su relación con las restantes categorías.

\section{DISCUSIÓN}

Este estudio pone de manifiesto la utilidad de los indicadores derivados del análisis de patentes para conocer la participación de hombres y mujeres en la actividad inventiva de un país e identificar tendencias temporales. Aunque la elaboración de este tipo de indicadores presenta algunas dificultades metodológicas que se describen brevemente a continuación, consideramos que su obtención de forma periódica es importante para poder realizar un seguimiento de la participación de las mujeres en la innovación y el desarrollo tecnológico.

\section{Dificultades metodológicas}

La falta de normalización en la información correspondiente al nombre de los inventores y solicitantes, así como de sus instituciones, es un importante problema que limita la obtención sistemática, automatizada y fiable de indicadores a partir del análisis de patentes. Una muestra de la preocupación que despierta la falta de normalización es la existencia de distintas iniciativas en la comunidad científica orientadas a la elaboración de algoritmos

Figura 5. Análisis de correspondencias múltiples.

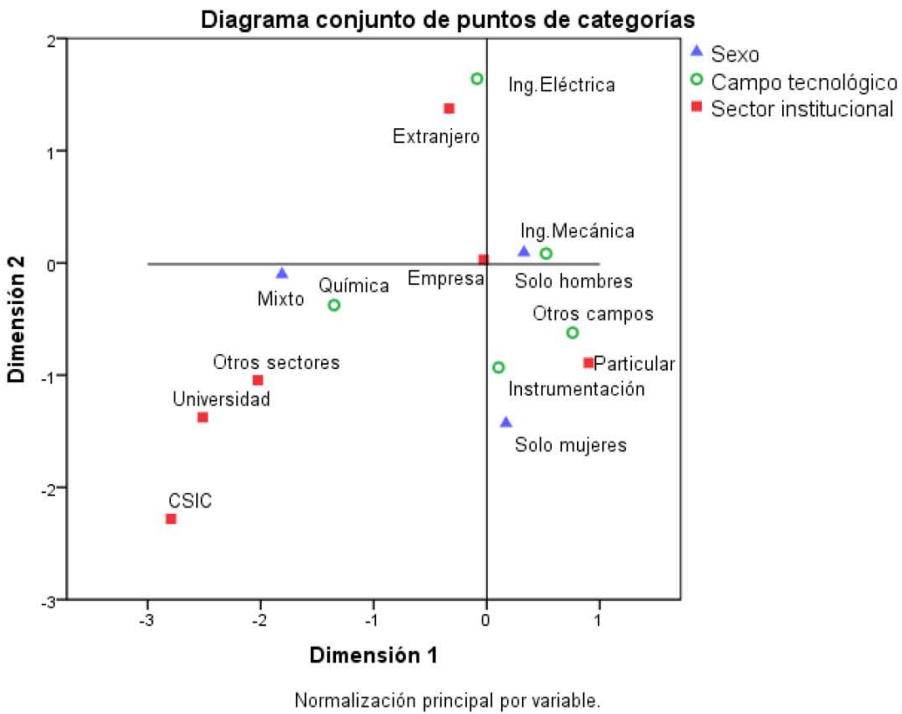


matemáticos para solventar el problema (veáse por ejemplo, el proyecto Academia Patenting in Europe (APE-INV) de la European Science Foundation, (http://www.esf.org/index.php?id=5800) centrado en la obtención de modelos matemáticos encaminados a la normalización de nombres de inventores y solicitantes). Aunque estas iniciativas están dando sus frutos, sería importante que los propios solicitantes de patentes y las oficinas correspondientes se implicaran en el uso de normalizado de nombres de instituciones, inventores y solicitantes.

En este trabajo se ha realizado una codificación semiautomática de los sectores institucionales, sin descender a instituciones o firmas y empresas. En lo que se refiere a los inventores, únicamente se ha identificado el sexo de cada ocurrencia, pero no se han normalizado todas las firmas de un mismo inventor, porque no resultaba indispensable para los fines de la investigación. El proceso de identificación del sexo de los inventores se vio facilitado por tratarse de inventores españoles, de forma que en muchos casos resultaba fácil deducir el sexo a partir del nombre del inventor. No obstante, esta identificación puede resultar muy laboriosa cuando se trabaja con inventores de distintos países y culturas. La inclusión del sexo de los inventores en los documentos de patentes facilitaría enormemente la obtención de indicadores por género.

\section{Actividad tecnológica por género}

En la actividad tecnológica española se observa un predominio claro de los inventores masculinos, de forma que sólo el $20 \%$ de las patentes analizadas cuentan con alguna mujer entre sus inventores. La contribución femenina es algo inferior, se sitúa en el $15 \%$, porque las mujeres colaboran con frecuencia con inventores masculinos. Esto se observa claramente a través de la participación: un $80 \%$ de las patentes están firmadas sólo por hombres, el $4 \%$ sólo por mujeres y el $16 \%$ por inventores de ambos sexos. La contribución femenina obtenida en nuestro estudio es sólo ligeramente superior a la descrita para España entre 2003-2005 (15\% vs $12.3 \%$ ) en un estudio sobre patentes solicitadas por 14 países a la EPO (Frietsch y otros, 2009), pero ese dato situaba a España, junto con Francia, a la cabeza de los países analizados en lo que a actividad femenina se refiere, presentando Alemania los menores valores. Otros estudios han señalado también estas diferencias entre países (ver por ejemplo, Naldi y otros, 2004; Giuri y otros, 2007) que se atribuyen a distintos factores como la estructura de los sistemas de ciencia y tecnología, el grado de inversión del sector privado en investigación y la presencia de mujeres en este último sector, que es el que muestra mayor orientación a patentar.

En nuestro análisis se observa que el CSIC y la universidad concentran la mayor actividad femenina (porcentaje de patentes con alguna mujer), mientras que los menores valores se detectan en las patentes solicitadas por empresas o particulares. Estos datos coinciden con los descritos en un estudio previo sobre las patentes solicitadas en la Oficina Española de Patentes (Mauleón y Bordons, 2010) y en parte es reflejo de la desigual distribución por sectores de las mujeres investigadoras en España, que tienen mayor presencia en la universidad que en el sector empresa (constituían el 38\% de los investigadores en las universidades frente a sólo un $27 \%$ en el sector empresa en 2006) (Comisión Europea, 2009). No obstante, la presencia femenina entre los inventores es también inferior a la esperada en función de su presencia entre los investigadores de los distintos sectores, lo que sugiere que no solo hay menos mujeres que hombres, sino que además ellas muestran una menor implicación en la solicitud de patentes. Estudios previos han señalado una menor tendencia de las mujeres a comercializar los resultados de su investigación (Stephan y El-Ganainy, 2007) y solicitar patentes, que se ha atribuido a distintos factores como son la menor financiación externa de su investigación, la menor relación de las mujeres investigadoras con la industria y una visión más "tradicional" de la investigación, que confiere mayor importancia a las publicaciones que a las patentes como resultados de la investigación (Ding y otros, 2006; Azoulay y otros, 2007). Según los trabajos anteriores, la dificultad en encontrar un equilibrio entre la investigación, la docencia y la transferencia de conocimientos al sector industrial y el temor a los posibles efectos negativos de esta última actividad sobre la carrera investigadora han sido algunas razones expresadas por distintas investigadoras entrevistadas para explicar su menor interés por patentar.

En cuanto a la presencia de la mujer en patentes de sectores de alto valor económico como son Biotecnología, TICs y Alta Tecnología; podemos decir que las mujeres están infra-representadas en los dos últimos campos, mientras que su actividad está por encima de la media en Biotecnología. En principio aquellas áreas de investigación de más reciente creación podrían suponer un espacio de avance para las mujeres, apoyadas en modelos de organización menos rígidos (Castaño y González Ramos, 2008; Whittington y Smith-Doerr, 2008), pero es posible que para aprovechar esta ventaja se requiera una cierta presencia femenina, como la que se da en las áreas de ciencias de la vida. La baja afinidad de las mujeres hacia algunos campos relacionados con las TICS y la Alta Tecnología, que se detecta desde la elección de los estudios universitarios, no facilita su integración en estos campos y su implicación en el desarrollo de nueva tecnología, aunque sería de gran interés favorecerla, dada su influencia sobre la competitividad y el desarrollo económico de los países.

El estudio a través del análisis de correspondencias permite ver una clara interrelación entre las variables participación, sector institucional y campo tecnológico. Así, las mujeres muestran una alta 
afinidad por la Química, y tienden a concentrarse en la universidad y el CSIC, sectores que a su vez están especializados en Química. La empresa y los particulares, que muestran alta especialización en Ingeniería Mecánica, presentan baja presencia de mujeres. Las diferencias por género en la selección de campos de estudio es un importante factor subyacente que contribuye a explicar esta desigual distribución de la actividad de hombres y mujeres (Comisión Europea, 2013), pero las decisiones individuales se ven también influidas por otros factores como el prestigio y el modelo organizativo de los distintos tipos institucionales (Whittington y Smith-Doerr, 2008). Esta mayor presencia de la mujer en el área de Química se detectó también en el análisis de patentes españolas (Mauleón y Bordons, 2010) y en otros países (Giuri, 2007; Ejermo y Jung, 2012; Hunt y otros, 2013).

La menor presencia de las mujeres en las patentes con un solo inventor podría explicarse por su mayor actividad en áreas donde predomina el trabajo en grupo o por su mayor tendencia a colaborar, independientemente del área. Nuestros datos muestran que incluso en las áreas temáticas más colaborativas (por ejemplo, Química), la presencia relativa de mujeres se incrementa con el tamaño de los equipos, lo que sugiere una mayor tendencia de las mujeres a colaborar. Este hallazgo podría relacionarse con la mayor orientación de las mujeres a desarrollar relaciones interpersonales (Bear y Woolley, 2011) y su preferencia por el trabajo colectivo frente al desarrollado de forma individual (Whittington y Smith-Doerr, 2008).

A lo largo del periodo, las mujeres tienden a incrementar ligeramente su participación como inventoras, de forma que el porcentaje de patentes con alguna mujer asciende desde el $16 \%$ en 1999 hasta el 24\% en 2007; igualmente aumenta la contribución femenina de un $10 \%$ a un $13 \%$, y el porcentaje de mujeres inventoras desde el $12 \%$ al $19 \%$. Este incremento se produce sobre todo en el último año del periodo y a expensas principalmente de patentes con inventores de ambos sexos. En relación a este último punto hay que mencionar que el creciente peso de las patentes con equipos mixtos puede ser considerado positivo desde dos puntos de vista: por un lado, porque facilita la incorporación de las mujeres a la actividad inventiva, pero también por los efectos positivos derivados de la diversidad de género en los grupos, que se ha relacionado con una mayor inteligencia colectiva y un mejor funcionamiento del grupo, que en último término puede facilitar la innovación (Bear y Woolley 2011).

El mayor incremento de la contribución femenina se produce en el sector público, y en particular en el CSIC y la universidad, que son los sectores que más incrementan su número de patentes en el periodo, aunque parten de cifras muy bajas de producción. El creciente interés de los gestores en España y otros países europeos por fomentar la llamada "tercera misión" de la universidad, es decir, las actividades de trasferencia y comercialización del conocimiento en forma de colaboración con la industria, solicitud de patentes y creación de spin-offs, puede ser un factor determinante de esta tendencia (Gulbrandsen y Slipersaeter, 2007; Azagra-Caro y Romero-de Pablos, 2009). Aunque la mayor contribución femenina se detecta en Química, el mayor incremento en esta contribución se produce en Instrumentación, lo que sugiere cierta diversificación del perfil temático de las mujeres.

Aunque nuestro estudio muestra solo una pequeña reducción de la brecha de género a lo largo del tiempo, sería interesante analizar posibles diferencias atendiendo a la edad de los investigadores. El estudio de Ding (Ding y otros, 2006) sobre una población de investigadores en ciencias de la vida pertenecientes al sector universitario en los Estados Unidos, puso de manifiesto la menor tendencia de las mujeres a patentar, pero también que estas diferencias se reducían entre las mujeres más jóvenes, lo que permitía esperar un mayor equilibrio entre géneros en los años futuros.

La baja presencia femenina entre los inventores españoles, que aumenta solo ligeramente a lo largo del periodo analizado, pone de manifiesto el interés de realizar estudios orientados a profundizar en los factores que contribuyen a la menor implicación de las mujeres en la solicitud de patentes, implementar medidas para reducir la brecha de género y producir indicadores periódicamente para hacer un seguimiento de la situación. Para favorecer la implicación de las mujeres en la solicitud de patentes se han sugerido en la literatura distintas vías de actuación, como por ejemplo incrementar los vínculos de las mujeres con el sector industrial, a ser posible desde la etapa doctoral y postdoctoral; promover una visión menos tradicional de la investigación, en la que la transferencia tecnológica tiene un importante papel y las patentes deben percibirse como un resultado legítimo de la actividad investigadora; disponer de oficinas de transferencia tecnológica en el sector académico para facilitar el contacto entre el mundo académico y la industria; y establecer una valoración positiva de las patentes en las evaluaciones del personal investigador. Aunque algunas de estas medidas son válidas para investigadores de ambos sexos, son especialmente importantes para las mujeres, que hoy por hoy requieren mayor apoyo institucional para compensar su menor integración en las redes profesionales (Ding y otros, 2006; Azoulay, y otros, 2007).

En definitiva, los indicadores basados en patentes desagregados por género constituyen una interesante herramienta para mejorar nuestro conocimiento sobre la presencia y participación femenina en actividades inventivas, permiten identificar posibles situaciones de desequilibrio por género, y 
son un apoyo a los gestores en sus tomas de decisión sobre medidas a implementar y evaluación de sus repercusiones. Estos indicadores pueden auxiliar en la formulación de políticas de género y de innovación tecnológica cuyo fin último es contribuir a la integración de la mujer investigadora en el sistema de ciencia y tecnología.

\section{AGRADECIMIENTOS}

Este trabajo se ha realizado en el marco de un proyecto financiado por el Ministerio de Trabajo y Asuntos Sociales (I+D+I 87/02).

\section{BIBLIOGRAFÍA}

Azagra-Caro, J.M.; Romero-De Pablos, A. (2009). Los determinantes institucionales de las patentes del Consejo Superior de Investigaciones Científicas: una aproximación histórica y una dialéctica con la Economía. Revista Española de Documentación Científica, vol.32 (2), 9-33. http://dx.doi.org/10.3989/redc.2009.2.680

Azoulay, P.; Ding, W.; Start, T. (2007). The determinants of faculty patenting behaviour: demographics or opportunities? Journal of Economic Behaviour and Organization, vol. 63 (4), 599-623. http://dx.doi.org/10.1016/j.jebo.2006.05.015

Balconi, M.; Breschi, S.; Lissoni, F. (2004). Networks of inventors and the role of academia: An exploration of Italian patent data. Research Policy, vol. 33 (1), 127-145.

Bear, J.B.; Woolley, A.W. (2011). The role of gender in team collaboration and performance. Interdisciplinary Science Reviews, vol. 36 (2), 146-153. http://dx.doi.org/10.1179/03080181 1X13013181961473

Benzécri, J.-P. (1973). L'Analyse des Donne'es. Vol. II. L'Analyse des Correspondances. Paris: Dunod.

Breschi, S.; Lissoni, F.; Montobbio, F. (2004). The scientific productivity of academic inventors: new evidence from Italian data. Economics of Innovation and New Technology, vol. 16 (2), 101-118. http://dx.doi. org/10.1080/10438590600982830

Busolt, U.; Kugele, K. (2009). The gender innovation and research productivity gap in Europe. International Journal of Innovation and Sustainable Development, vol. 4 (2-3), 109-122. http:// dx.doi.org/10.1504/IJISD.2009.028066

Castaño, C.; González Ramos, A.M. (2008). La disparidad entre la participación y la posición de las mujeres en la investigación TIC: El caso del Plan Nacional de I+D+i. En: González Hermoso de Mendoza, A.; Morcillo Ortega, P. Coord. El Plan Nacional de I+D+i (2008-2011) a examen. Revista madri+d. Monografía 21. Junio 2008. Pp. 118-126.

Comisión Europea (2000). Science Policies in the European Union: Promoting excellence through mainstreaming gender equality. A report from the ETAN Network on Women and Science. Luxemburgo: Comisión Europea. ISBN 92-8288682-4.

Comisión de las Comunidades Europeas. (2001). Las mujeres y la ciencia: utilidad de la dimensión del género para impulsar la reforma científica. Documento de Trabajo de los servicios de la Comisión SEC (2001) 771, Bruselas. Accesible en: ftp://ftp.cordis.europa.eu/pub/improving/ docs/g_wo_sec771_es_200101.pdf

Comisión Europea (2009). She Figures 2009. Statistics and Indicators on Gender Equality in Science. European Commission. DirectorateGeneral for Research, Directorate C-Science and Society, Unit C.4-Women and Science. Brussels.

Comisión Europea (2010). Stocktaking 10 years of "Women in Science" policy by the European Commission 1999-2009. Luxemburgo: Comisión Europea. ISBN 978-92-79-13910-9.

Comisión Europea (2013). She Figures 2012. Gender in Research and Innovation. Bruselas: Comisión Europea. http://ec.europa.eu/research/ science-society/document_library/pdf_06/shefigures-2012_en.pdf

Delixus Inc. (2012). Intellectual Property and Women Entrepreneurs. Report to the National Women's Business Council. En: http://nwbc. gov/sites/default/files/IP\%20\&\%20Women\%20 Entrepreneurs.pdf

Dewandre, N. (2002). European strategies for promoting women in science. Science, vol. 295, 278-279. http://dx.doi.org/10.1126/science. 1063487

Ding, W.W.; Murray, F.; Stuart, T.E. (2006). Gender differences in patenting in the academic life sciences. Science, vol. 313 (5787), 665-687. http://dx.doi.org/10.1126/science.1124832

Ejermo, O.; T. Jung (2012), Demographic patterns and trends in patenting: Gender, age, and education of inventors, CIRCLE Working Paper, WP2012/05

Frietsch, R.; Haller, I.; Funken-Vrohlings, M.; Grupp, H. (2009). Gender-specific patterns in patenting and publishing. Research Policy, 38/4, 590-599. pol.2009.01.019

Giuri, P.; Mariani, M.; Brusoni, S.; Crespi, G.; Francoz, D.; Gambardella, A.; Garcia-Fontes, W.; Geuna, A.; Gonzales, R.; Harhoff, D.; Hoisl, K.; Le Bas, C.; Luzzi, A.; Magazzini, L.; Nesta, L.; Nomaler, Ö.; Palomeras, N.; Patel, P.; RomaneIli, M.; Verspagen, B. (2007). Inventors and invention processes in Europe: Results from the PatVal-EU survey. Research Policy, vol. 36, n. 8, 1107-1127. http://dx.doi.org/10.1016/j.respol.2007.07.008

Gulbrandsen, M.; Slipersaeter, S. (2007). The third mission and the entrepreneurial university model. En: Bonaccorsi, A.; Daraio, C. Eds. Universities and strategic knowledge creation. 
Specialization and performance in Europe. Cheltenham (UK), Northampton (MA): Edward Elgar. Pp.112-143.

Hunt, J.; Garant, J.-P.; Herman, H.; Munroe, D. J. (2013). Why are women underrepresented amongst patentees? Research Policy 42: 831-843. http://dx.doi.org/10.1016/j.respol.2012.11.004

Lascuraín, M.L.; Madera-Jaramillo, M.J.; Ortoll, E.; Sanz-Casado, E. (2010). Capacidad innovadora de la Comunidad de Madrid a partir de las patentes concedidas entre 1996 y 2007. Revista Española de Documentación Científica, vol.33 (3), 458-479. http://dx.doi.org/10.3989/ redc. 2010.3 .761

Mauleón, E.; Bordons, M. (2010). Male and female involvement in patenting activity in Spain. Scientometrics, vol. 83 (3), 605-621. http:// dx.doi.org/10.1007/s11192-009-0131-x

Mauleón, E.; Daraio, C.; Bordons, M. (2014). Exploring gender differences in patenting in Spain. Research Evaluation, vol. 23 (1), 62-78. http:// dx.doi.org/10.1093/reseval/rvt030

Morillo, F.; Aparicio, J.; González-Albo, B.; Moreno, L. (2013). Towards the automation of addresses identification. Scientometrics, vol. 94 (1), 207224. http://dx.doi.org/10.1007/s11192-0120733-6

Morgan, R.P.; Kruytbosch, C.; Kannankytty, N. (2001). Patenting and invention activity of U.S. scientists and engineers in the academic sector: comparisons with industry. Journal of Technology Transfer, vol.26, 173-183. http://dx.doi. org/10.1023/A: 1007856800497

Murray, F.; Graham, L. (2007). Buying science and selling science: gender differences in the market for commercial science. Industrial and Corporate Change, vol.16 (4), 657-689. http://dx.doi. org/10.1093/icc/dtm021

Naldi, F.; Luzi, D.; Valente, A.; Vannini, P. (2004). Scientific and technological performance by gender. In Moed, H.F.; Glanzel, W. and Schmoch, U. (Eds.). Handbook of Quantitative Science and
Technology Research. Kluwer Academic Publishers, Dordrecht, pp. 299-314.

Nesta, P.; Patel, P. (2004). National patterns of technology accumulation: use of patent statistics. In: Moed, H. F.; Glanzel, W. ; and Schmoch, U., (Eds.). Handbook of Quantitative Science and Technology Research. Kluwer Academic Publishers, Dordrecht, pp. 531-551.

OEPM (2009). Titulares de patentes nacionales, según el titular, desde el año 1964 al 2008. Servicio de Estadísticas y Estudios. Accesible en: http://www.oepm.es/es/sobre_oepm/actividades_estadisticas/estadisticas/estudios_estadisticos/index.html

OMPI. (2008). Informe de la OMPI sobre patentes. Estudio estadístico. Organización Mundial de la Propiedad Intelectual. [http://www.wipo. int/export/sites/www/freepublications/es/patents/931/wipo_pub_931_2008.pdf]

Schild, I. (1999). A regional patent study to investigate inventive activity in East Gothia. University of Linkoping. Working paper n. 207.

Schmoch, U. (2008). Concept of a technology classification for country comparisons. Final report to the World Intellectual Property Organisation (WIPO). [http://www.wipo.int/edocs/mdocs/ classifications/en/ipc_ce_41/ipc_ce_41_5-annex1.pdf]

Stephan, P.E.; El-Ganainy, A. (2007). The entrepreneurial puzzle: explaining the gender gap. Journal of Technology Transfer, vol. 32, 475487. http://dx.doi.org/10.1007/s10961-0079033-3

Whittington, K.B.; Smith-Doerr, L. (2005). Gender and commercial science: women's patenting in the Life Sciences. Journal of Technology Transfer, vol. 30 (4), 355-370. http://dx.doi. org/10.1007/s10961-005-2581-5

Whittington, K.B.; Smith-Doerr, L. (2008). Women inventors in context. Disparities in patenting across academia and industry. Gender and Society, vol. 22 (2), 194-218. http://dx.doi. org/10.1177/0891243207313928 